Research Article

\title{
Development of a Novel Sensor System Based on Magnetic Microspheres to Detect Cardiac Troponin T
}

\author{
Junrong Zhang, ${ }^{1}$ Hongxiang Liu, ${ }^{1}$ Baofeng Xu, ${ }^{2}$ Sijun Huang, ${ }^{1}$ Rui Liu, ${ }^{3}$ Jinming Zhu, ${ }^{3}$ \\ Yi Guo $\mathbb{D}^{1}{ }^{1}$ and Li Xu ${ }^{1}{ }^{1}$ \\ ${ }^{1}$ Key Laboratory for Molecular Enzymology and Engineering, The Ministry of Education, National Engineering Laboratory for \\ AIDS Vaccine, School of Life Sciences, Jilin University, Changchun 130012, China \\ ${ }^{2}$ Department of Neurosurgery, The First Hospital of Jilin University, Changchun 130021, China \\ ${ }^{3}$ China-Japan Union Hospital of Jilin University, Changchun 130033, China
}

Correspondence should be addressed to Yi Guo; guoyi@jlu.edu.cn and Li Xu; xuli@jlu.edu.cn

Received 2 September 2020; Revised 18 November 2020; Accepted 20 November 2020; Published 3 December 2020

Academic Editor: Peter Foot

Copyright ( $\odot 2020$ Junrong Zhang et al. This is an open access article distributed under the Creative Commons Attribution License, which permits unrestricted use, distribution, and reproduction in any medium, provided the original work is properly cited.

Acute myocardial infarction (AMI) causes irreversible injury to cardiomyocytes in a short time and may result in various complications, severely threatening patient safety. Therefore, it is necessary to predict the possibility of AMI in the prophase. Prognostic detection of biomarkers that specifically reflect myocardial damage in a patient's blood has become an essential mediating measure to prevent the serious occurrence of AMI. The present study is aimed at exploring a novel sensing system with high specificity and precision based on magnetic microspheres developed to detect cardiac troponin $\mathrm{T}$ (cTnT), which is the most specific diagnostic marker for AMI in cardiovascular diseases. Naive human cTnT protein in serum samples and antigens on functional magnetic microspheres will competitively bind with limited specific antibodies. After rapid removal of heterogeneous elements in the sera using a magnetic separator, fluorescein isothiocyanate-labeled immunoglobulin $G$ is added to react with specific antibodies on the magnetic microspheres. Then, a flow cytometer is used to collect signals of different fluorescence intensities. The results show that the method is characterized by economy, high accuracy, and novelty. It can be used for the detection of cTnT in blood at $1.7-106.1 \mathrm{ng} / \mathrm{mL}$, with a detection limit of $0.5 \mathrm{ng} / \mathrm{mL}$. Thus, the proposed sensor improves the accuracy and efficiency of diagnosis before clinical deterioration of AMI.

\section{Introduction}

Acute myocardial infarction (AMI) is a threat to human health that causes disability and is characterized by high morbidity. Therefore, establishing a rapid and accurate diagnostic testing method at an early stage will reduce mortality, disability, and high costs [1-3]. So far, three techniques have been used in combination to confirm diagnosis: (a) clinical history examination of patients with ischemic chest pain, (b) dynamic evolution of an electrocardiogram (ECG) [4], and (c) detection of the fluctuation of concentration of serum biomarkers in myocardial necrosis or heart failure [5]. The first method is beyond the scope of this discussion, and the second method relies on experienced clinicians but has many uncertainties. This creates limitations in clinical and laboratory applications. According to previous reports, $25 \%$ of patients have no obvious typical symptoms and more than $50 \%$ of patients have no ECG changes. This has created obstacles to definite diagnosis in the development of ECG for AMI diagnostic purposes [6]. In comparison, serum index detection has been widely used in the diagnosis of AMI due to its simple detection process, high sensitivity, and strong specificity $[7,8]$.

Cardiac troponin (cTn) is a unique protein in cardiomyocytes, and very little of it is released in the serum of healthy people [9]. cTn includes three different subunits: $c \mathrm{TnT}, \mathrm{cTnI}$, and TnC. It acts as a regulatory protein in myocardial contraction and is released into the bloodstream when the heart is damaged. In addition, the detection of cTn has high sensitivity and specificity and may substitute traditional cardiac enzymes as a diagnostic indicator of AMI [10-14]. Therefore, the fluctuation of cTnT content is regarded as an important 
indicator of AMI diagnosis by the European and American College of Cardiology [15].

The current methods for detecting cTnT are mainly electrochemiluminescence (ECL) and enzyme-linked immunosorbent assay (ELISA) [16]. However, ECL requires skilled operators, has high reagent consumption, and may exhibit cross-reactivity with toxins that are similar in structure or with matrix components [17]. Furthermore, ELISA is prone to false positives that negatively affect the test results [18]. Therefore, a low-cost, easy-to-operate, accurate, and specific measure has become an indispensable technology in contemporary medical treatment.

Compared with these conventional analytical techniques, the cytometric bead array (CBA) assay can provide high sensitivity and selectivity for simultaneous high-throughput detection of multiple targets [19-21]. It is particularly sensitive to micromolecules owing to the competition in the fluid stream [22]. As an important testing candidate, magnetic microspheres may provide a more efficient, automated, parallelizable, and controllable technique for separating multiple targets [23, 24]. Magnetic microspheres are a new type of carrier that can be conjugated with different kinds of probe molecules. They provide the advantages of assay automation and improved sensitivity and specificity detection [25]. Moreover, magnetic separation technology allows low shear stress, and will not adversely affect the conformation and function of proteins during the antigen-antibody binding and washing process. Based on the properties of magnetism, magnetic separation has been applied extensively in many fields [26-28].

In this study, based on the principle of direct competition, magnetic microspheres were used to develop a practical system for detecting cardiac troponin $\mathrm{T}$ (cTnT). The system has advantages of simplicity, rapidity, and economy and is environmentally benign; it requires only a single analyte-specific antibody to achieve high accuracy and specificity. A schematic representation of a cTnT detection method based on magnetic microspheres is shown in Figure 1. Functional microspheres were constructed via covalent bonding between carboxyl magnetic spheres and cTnT in an Eppendorf (EP) tube. Then, the microspheres and cTnT-specific antibodies were added to the serum samples. The possible target (cTnT) in the sera and the cTnT on the surface of functional microspheres would compete with the limited specific recognition units on antibodies for binding. Through competitive binding and reaction, magnets were introduced to separate and enrich the magnetic microspheres for effective magnetic isolation. Then, fluorescein isothiocyanate- (FITC-) labeled goat anti-rabbit immunoglobulin $\mathrm{G}$ (IgG) fluorescent probes were added to bind with the captured antibody on the surface of the microspheres. After magnetic separation, the real-time fluorescence intensity of the reporter probes on the surface of the microspheres was determined by a sensitive flow cytometer, which performed a qualitative and quantitative analysis of cTnT in sera. A capability coupling analysis method revealed that the real-time fluorescence intensity was negatively correlated with the real quantitation analytes in the tested sample.

\section{Materials and Methods}

2.1. Experimental Materials and Reagents. In this work, all the reagents were analytically pure. 2-(N-Morpholino)ethanesulfonic acid (MES) was purchased from GEN-VIEW SCIENTIFIC INC. Tween-20 was obtained from Amresco (Houston, USA). 1-Ethyl-3-(3-dimethylaminopropyl)carbodiimide hydrochloride (EDC) and N-hydroxysuccinimide (NHS) were obtained from Sigma-Aldrich (MO, USA). Natural human cTnT protein was purchased from Abcam (Cambridge, UK). Rabbit anti-cTnT and goat anti-rabbit IgG/FITC (IgG-FITC) were obtained from Beijing Bioss Antibodies Co., Ltd. (Beijing, China). Carboxyl-modified magnetic microspheres (a mean diameter particle of $4.5 \mu \mathrm{m}$ ) were purchased from BaseLine Chromtech Research Centre (Tianjin, China).

MES (50 mM) was adjusted to $\mathrm{pH} 6.0$ and $\mathrm{pH} 5.0$ to serve as an activation and coupling buffer, respectively. $0.1 \%(v / v)$ Tween-20 was dissolved in phosphate buffer saline (PBS, $10 \mathrm{mM}, \mathrm{pH}$ 7.4) to prepare PBST washing buffer. PBS supplemented with $0.1 \%(w / v)$ bovine serum albumin (BSA), $0.01 \%$ $(v / v)$ Tween-20, and $0.05 \%(w / v) \mathrm{NaN}_{3}$ (PBSBTN) was used as a storage buffer. The activation buffer MES (50 mM MES, $\mathrm{pH}$ 6.0) and the coupling buffer (50 mM MES, pH 5.0) were prepared before use.

\subsection{Magnetic Microspheres Covalently Coupled with cTnT.} The immobilization of cTnT protein on the surface of the carboxylic magnetic spheres $\left(1.07 \times 10^{6} / \mathrm{mL}\right)$ was performed in a $2 \mathrm{~mL}$ EP tube. Activating the carboxyl group involved washing it using the activation buffer MES and the coupling buffer. The activation buffer $(200 \mu \mathrm{L})$ was added to the magnetic spheres $(100 \mu \mathrm{L})$ and subjected to vortex oscillation for $30 \mathrm{~s}$. Then, the buffer was suspended for $5 \mathrm{~min}$ and subjected to magnetic separation. Additionally, the microspheres were resuspended in $160 \mu \mathrm{L}$ of coupling buffer, $20 \mu \mathrm{L}$ of EDC (0.32 mM; dissolved in deionized water), and $20 \mu \mathrm{L}$ of NHS (0.33 mM; dissolved in deionized water). After incubation for $30 \mathrm{~min}$, the microspheres were separated by a magnetic separator and the supernatant was carefully discarded and washed three times with the coupling buffer. Then, the cTnT protein was added to the coupling buffer $(1 \mathrm{~mL})$ and incubated with the purified microspheres $(2 \mathrm{~mL})$ at $37^{\circ} \mathrm{C}$ for $2 \mathrm{~h}$ with shaking. After separating the coupled microspherecTnT conjugates using a magnetic separator, the supernatant was carefully discarded and the precipitate was washed 2 times. Next, $500 \mu \mathrm{L}$ of blocking buffer was added into EP tubes and incubated at body temperature for $2 \mathrm{~h}$. Finally, the microsphere-cTnT conjugates were enriched with a magnetic separator and washed 3 times using PBST. Then, the conjugates were stored in $1000 \mu \mathrm{L}$ PBSBTN at $4^{\circ} \mathrm{C}$ in the dark for subsequent experiments.

2.3. Detection of $c \operatorname{Tn} T .100 \mu \mathrm{L}$ of the serum sample with cTnT or cTnT standard dilution, $8 \mu \mathrm{L}$ of magnetic microspherecTnT conjugate, and $100 \mu \mathrm{L}$ of specific cTnT antibodies were added into an EP tube. The system was mixed using vortex oscillation and incubated for $150 \mathrm{~min}$. After magneticenrichment and washing 3 times, $100 \mu \mathrm{L}$ of goat anti-rabbit 


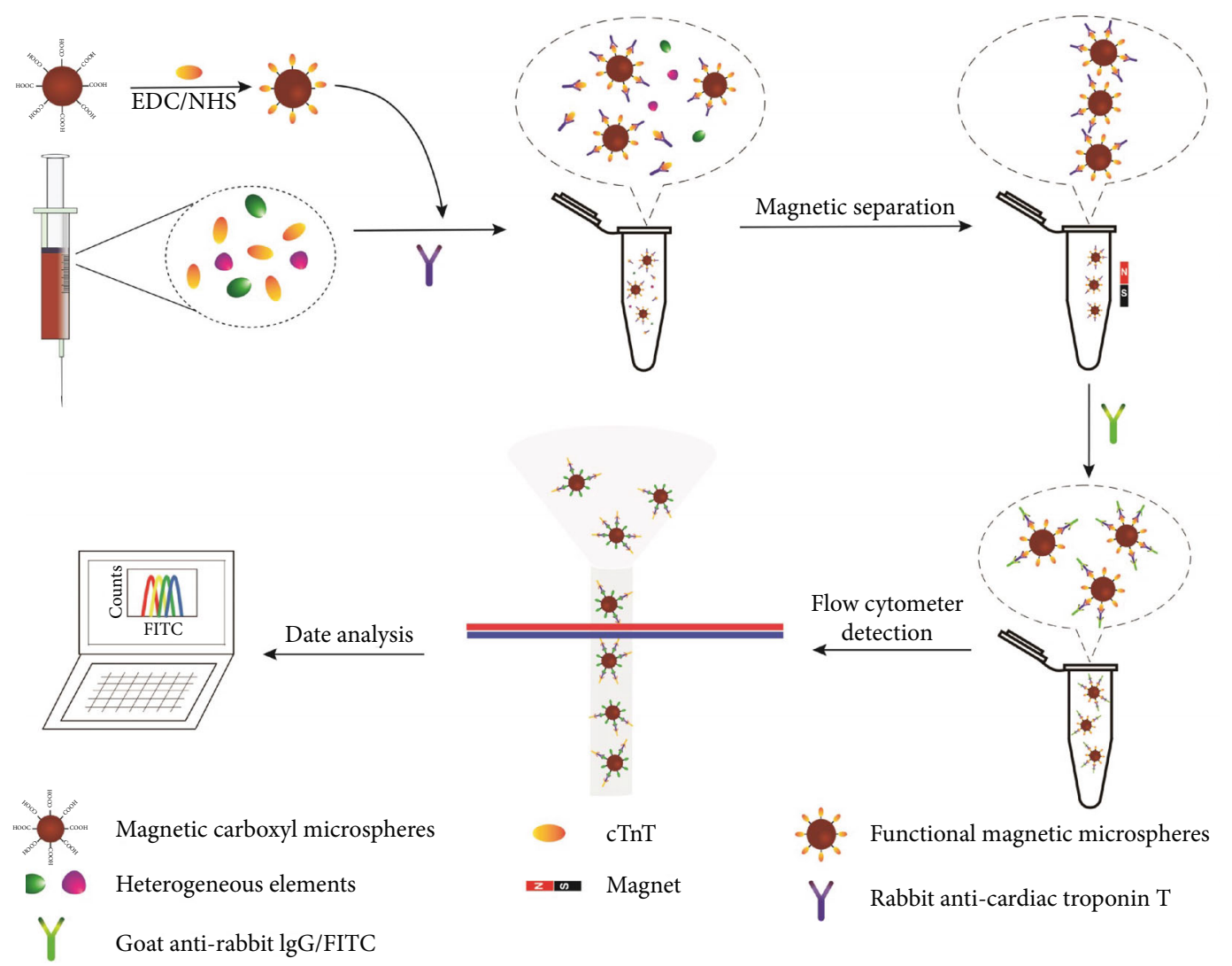

FIgURE 1: Schematic representation of a cTnT detection method based on magnetic microspheres.

IgG/FITC was added and incubated for $1 \mathrm{~h}$. All the incubation samples were shaken at $37^{\circ} \mathrm{C}$ in the dark. The separation step took about $2 \mathrm{~min}$, and after the washing buffer was removed, PBS ( $\mathrm{pH}$ 7.4) was added into the system and fluorescent detection was performed in dedicated FACS tubes. Due to the quenching of organic fluorescence, the measurement had to be taken as soon as possible.

A flow cytometer was used to measure forward scattering (FSC), side scattering (SSC), and FITC signal (FL1) sources of each magnetic sphere. The functional spheres were mixed with serum diluent or a buffer containing cTnT. After incubation, fluorescently labeled probes were added to form a sandwich structure with florescence. The intensity signals of FSC, SSC, and FITC were negatively correlated with the concentration of cTnT.

2.4. Data Analysis. To optimize data from the FACSCalibur flow system, mean fluorescence intensity (MFI) is a desirable parameter in various severe conditions. The MFI computational formula was followed; it accurately reflects the concentration of cTnT in a sample or standard dilution solution, and the average fluorescence intensity (MFI) and binding reaction can be used as measurement indicators as shown in the following:

$$
\mathrm{MFI}=\frac{\left[S_{n}-S_{\min }\right]}{n} .
$$

In the formula, MFI is the average fluorescence intensity of each magnetic sphere-cTnT binding to the fluorescent probe under the same control conditions, $S_{n}$ is the fluorescence signal detected in the sample or standard, and $n$ represents the number of samples. Moreover, $S_{\min }$ is the background fluorescence signal intensity; the magnetic sphere-cTnT do not bind to any specific antibody or IgG/FITC.

$$
R(\%)=\frac{S_{n}-S_{\min }}{S_{\max }-S_{\min }},
$$

where $R$ is the specific rabbit anti-cTnT antibody with a fluorescent binding reaction, $S_{n}$ is the intensity of the fluorescence signal obtained in the sample or standard, $S_{\min }$ is the background fluorescence signal intensity and the magnetic sphere-cTnT do not bind to specific antibodies or IgG/FITC, and $S_{\max }$ is the fluorescence signal intensity which of maximum anti-cTnT antibody coupled with functional magnetic spheres. In order to reduce possible errors in the statistical analysis, three independent experiments were performed on each sample.

$$
Y=A_{2}+\frac{\left(A_{1}-A_{2}\right)}{1+\left(X / X_{0}\right)^{P}}
$$

In the fitted curve formula, $X$ is the concentration in the 


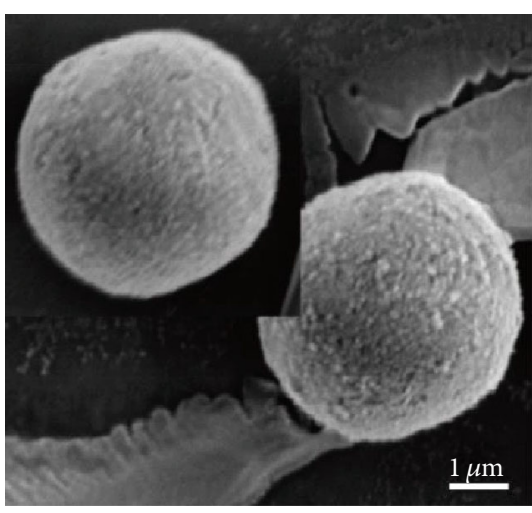

(a)

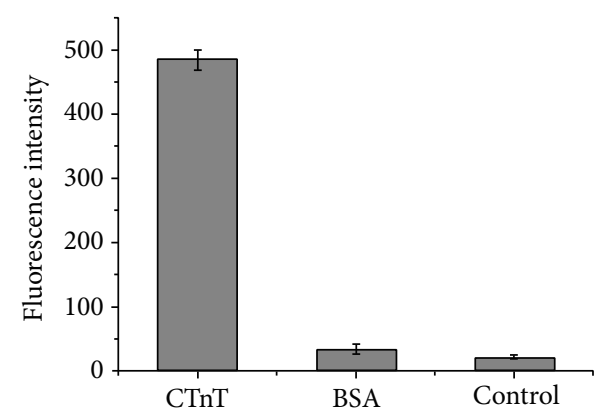

(b)

Figure 2: (a) SEM images of magnetic microspheres (in the upper left corner) and magnetic microspheres-cTnT (in the center). (b) Biological characteristics of microspheres-cTnT and microspheres-BSA.

unknown samples and $Y$ is the binding response value. $A_{1}$, $A_{2}, X_{0}$, and $p$ are constants.

\section{Results and Discussion}

3.1. Magnetic Microspheres Modified with cTnT. Magnetic microspheres were modified with the target protein cTnT to make them compete with anti-cTnT for binding. Figure 2(a) shows SEM (scanning electron microscope) images of natural (in the upper left corner) and functional (in the center) magnetic microspheres with smooth surfaces and rough textures, respectively, indicating that cTnT successfully adhered to the surface of the magnetic microspheres. Furthermore, biological analysis experiments verified the combination. The natural magnetic microspheres, microspheres-cTnT, and microspheres-BSA (magnetic microspheres coupled with BSA) were incubated with anti-cTnT followed by incubation with goat anti-rabbit IgG/FITC. As shown in Figure 2(b), only cTnT coupled with magnetic microspheres showed obvious fluorescence intensity, indicating that cTnT was successfully connected to the magnetic microspheres and had a high affinity for the corresponding antibodies.

3.2. Condition Optimization. As the diversity of complex element structures seriously threatens the sensitivity and specificity of the detection system, each condition was reexplored through experiments. In the general optimization stage, samples and standard targets were not added to the measurement, and the MFI of the FITC-IgG fluorescent reporter probe on the surface of the magnetic microspheres was used as an evaluation index.

The carboxyl groups on the magnetic particles allowed the covalent binding between cTnT and the microparticles to stabilize the structure of the functional magnetic microspheres. Furthermore, the higher concentration of coated antibodies added to the functional magnetic microspheres provided improved ability to capture the target. However, excessive coating antibodies may cause steric hindrance on the coating surface [29]. In order to optimize the concentration of rabbit anti-cTnT in the analysis system, $8.6 \times 10^{3}$ microspheres were used for this work. Therefore, $8 \mu \mathrm{L}$ of magnetic microspheres and $100 \mu \mathrm{L}$ of anti-cTnT at 25, 50, 100,200 , and $400 \mathrm{ng} / \mathrm{mL}$ were selected. Theoretically, the MFI of magnetic microspheres is positively correlated with the concentration of cTnT antibodies and is the optimal detection condition until the amount of anti-cTnT reaches saturation. Figure 3(a) shows that the MFI collected by the flow cytometer increased logarithmically until the concentration of anti-cTnT was $200 \mathrm{ng} / \mathrm{mL}$, indicating that this was the ideal detection concentration.

Goat anti-rabbit IgG/FITC on the surface of coded magnetic microspheres served as signal molecules and was found to be negatively correlated with cTnT content in the sample or standard. An appropriate amount of IgG-FITC is essential for optimal fluorescence intensity and accurate quantification; therefore, different multiples of dilution concentrations were used. Figure 3(b) shows that the fluorescent intensity of coded microspheres gradually increased with increasing concentrations of antirabbit IgG/FITC with $0.8,1.0,1.3,2,4$, and $100 \mu \mathrm{g} / \mathrm{mL}$ or dilution ratios of $1: 2500,1: 2000,1: 1500,1: 1000$, $1: 500$, and $1: 20$, respectively. However, MFI of FITCIgG fluorescent reporter probes on the surface of magnetic microspheres reached saturation as the concentration of IgG-FITC became $4 \mu \mathrm{g} / \mathrm{mL}$ or a ratio of dilution of $1: 500$. This shows that almost all anti-cTnT on the surface of the magnetic microspheres combined with IgGFITC in the solution to guarantee optimal conditions.

In addition, incubation time is a crucial parameter that influences the combination of anti-cTnT and IgG-FITC with microspheres-cTnT and coded microspheres-cTnT, respectively. According to the antigen-antibody reaction theory, sufficient incubation time can increase the density of anticTnT on the microsphere surfaces. This has been proven with general western blot. However, considering the high sensitivity of FITC fluorescein to light and the stability of goat anti-rabbit IgG-FITC and rabbit anti-cTnT as well as to save time and reduce the fluorescence quenching of FITC, we obtained the results shown in Figure 3(c). Fluorescence intensity increased with incubation time between 30 and 150 min till the optimal time for anti-cTnT to couple with 


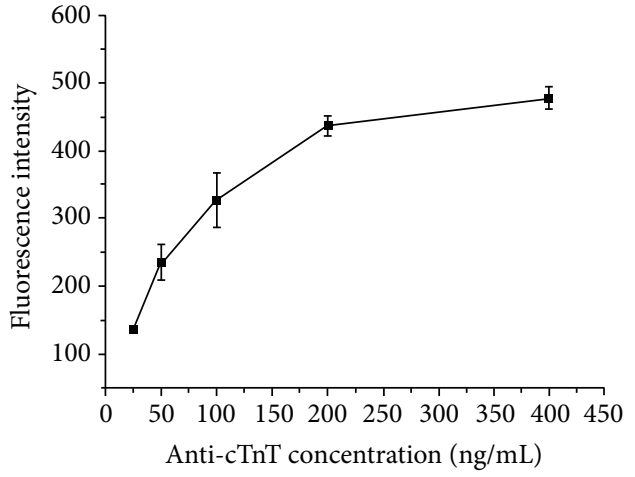

(a)

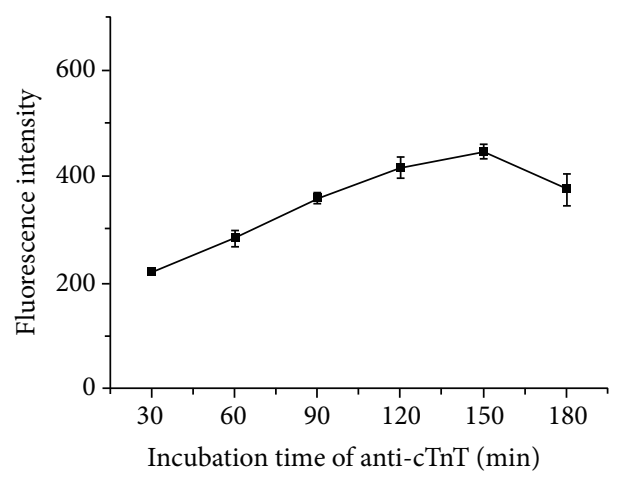

(c)

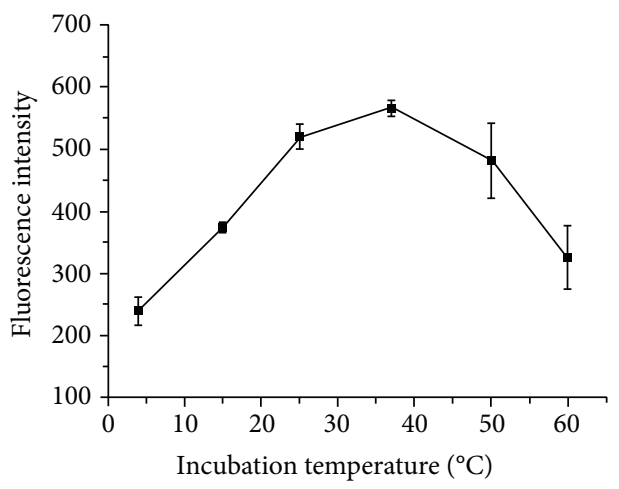

(e)

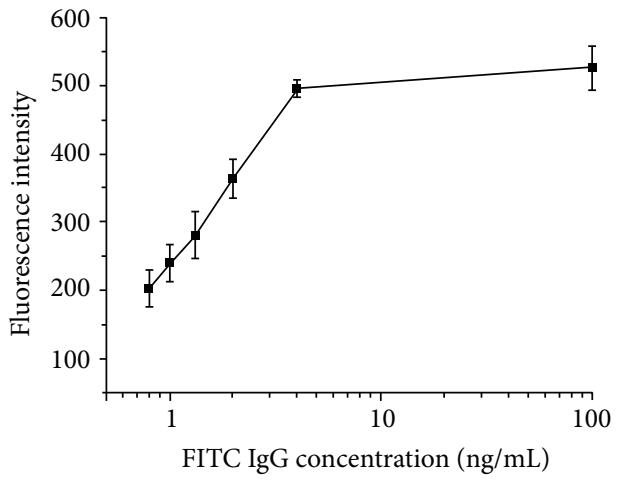

(b)

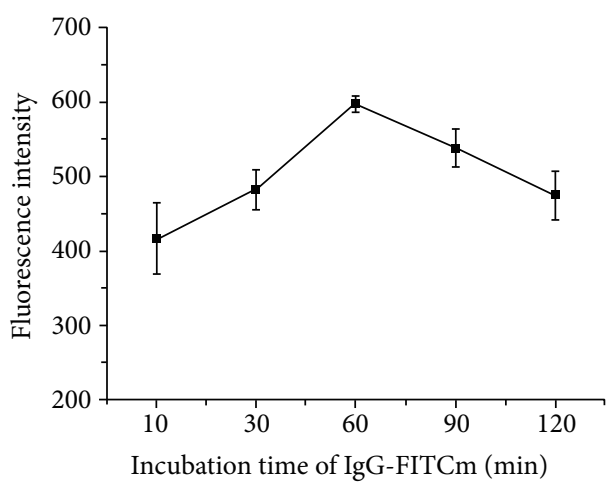

(d)

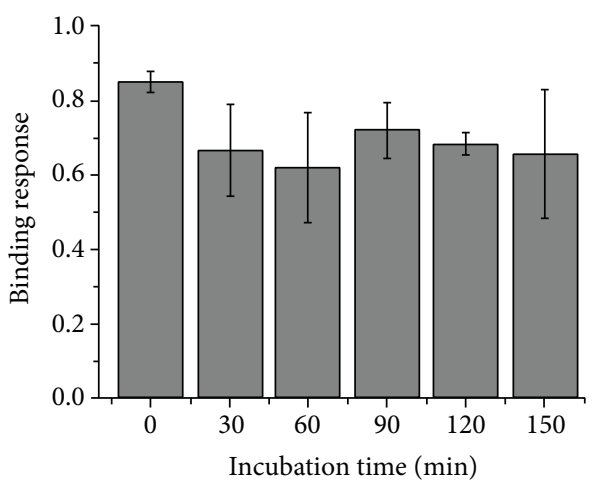

(f)

FIGURE 3: (a) MFI of magnetic microspheres--cTnT incubated with different concentrations of anti-cTnT at 25, 50, 100, 200, and 400 ng/mL, respectively. (b) MFI of coded conjugations incubated with different concentrations of IgG-FITC, i.e., $0.8,1.0,1.33,2.0,4.0$, and 100 $\mu \mathrm{g} / \mathrm{mL}$ or at dilution ratios of $1: 2500,1: 2000,1: 1500,1: 1000,1: 500$, and $1: 20$, respectively. (c) MFI of magnetic microspheres-cTnT incubated with anti-cTnT for 30, 60, 90, 120, and 150 minutes. (d) MFI of coded conjugate incubated with different IgG-FITC concentrations for 10, 30, 60, 90, and 120 minutes. (e) Incubation temperatures of the reaction system at a range of 4, 15, 25, 37, 50, and $60^{\circ} \mathrm{C}$. (f) Binding response of cTnT in the samples or standard with anti-cTnT at the first reaction step.

magnetic microspheres was reached. Thus, 150 min was chosen as incubation time in subsequent experiments.

The optimal time for IgG-FITC binding is shown in Figure 3(d); all fluorescent antibodies bind to the magnetic microsphere $\mathrm{c} \operatorname{Tn} \mathrm{T}$ at a maximum fluorescence intensity of $60 \mathrm{~min}$. The incubation temperature is another important parameter for antigen-antibody immune recognition in vitro. The incubation temperature easily affects the fluorescence intensity or quantitative accuracy of FITC on the surface of magnetic microspheres. As shown in Figure 3(e), fluorescence intensities on the surface of the microspheres changed with increasing incubation temperatures between 10 and $60^{\circ} \mathrm{C}$. The fluorescence intensity of conjugates increased with increasing incubation temperature from $10^{\circ} \mathrm{C}$ to $37^{\circ} \mathrm{C}$, followed by a decreasing trend after $37^{\circ} \mathrm{C}$. Therefore, the appropriate incubation temperature for anticTnT coupled with magnetic microspheres-cTnT and IgGFITC linked with coded microspheres-cTnT was $37^{\circ} \mathrm{C}$.

The cTnT in the samples or standard coupled with anticTnT through electrostatic attraction, van der Waals forces, hydrogen bonds, hydrophobic forces, and other less prominent stable combination methods. The noncovalent bond 
combination showed a certain degree of dependence on incubation time, and cTnT in the samples or standards combined with anti-cTnT in the solution. Appropriate incubation time could increase the coupling efficiency of all cTnT and anticTnT in the sample and standard solution, and the remaining anti-cTnT would bind to the cTnT on the surface of the magnetic microspheres thereby achieving high sensitivity and accurate quantification. In order to evaluate the binding reaction of cTnT and anti-cTnT in the solution, $100 \mathrm{ng} / \mathrm{mL}$ of cTnT in PBS (10 mM; pH 7.4) was selected. As shown in Figure $3(\mathrm{f})$, the maximum binding reaction incubation time was $0 \mathrm{~min}$ up to $84.97 \%$. It is worth mentioning that direct and indirect competitions are used in a series of detection methods. Indirect competition incubates the cTnT in the samples or standards with anti-cTnT between 30 and $150 \mathrm{~min}$ before adding the magnetic microspheres-cTnT to the system. The direct competition mode involved the addition of functional magnetic microspheres, cTnT, and antibodies in serum samples or standards to the EP tube at the same time of the reaction. The results indicate that direct competition was an optimal method to decrease the influence of unstable antigen-antibody binding in the solution on lowering the SD (Standard Deviation) value.

3.3. Performance Verification. Fluorescence signals from the IgG-FITC attached to the magnetic microspheres were used to quantitatively determine analyte concentration. Figure 4(a) shows scatter plots (SSC-H vs. FSC-H) which helped to separate permitted or distinguished particle sizes. As shown in the scatter plots (FL1-H vs. FSC-H), $100 \mathrm{ng} / \mathrm{mL}$ of $\mathrm{CTnT}$ in the standard was used in the established detection system. After prior optimization work, the target cTnT dissolved into PBS was quantified using the established magnetic microsphere-based detection assay. According to the principle of direct competition and antigen-antibody reaction, the anti-cTnT adhering to the surface of the microspheres would reduce with increasing cTnT levels in the standard. Figure 4(c) shows a histogram of the magnetic microspheres, and the target concentration-responsive signal change in FL1 can be clearly observed. The binding reaction of the microspheres in each gradient experiment was calculated to quantitatively analyze the target protein in the samples or standards. Figure 4(d) shows binding response (R) variation with different concentrations of cTnT. For the standard curve, values of 11 triplicate gradient concentrations of cTnT dissolved in PBS at concentrations of 0.1, 0.2, $0.5,1,2,5,10,20,50,100$, and $150 \mathrm{ng} / \mathrm{mL}$ dissolved in PBS are used. The binding reaction of magnetic microspherescTnT and anti-cTnT showed an "S" curve that gradually decreased with an increase in cTnT concentration. The target concentration decrease was particularly pronounced in the $1-100 \mathrm{ng} / \mathrm{mL}$ concentration range.

3.4. Accuracy. Accuracy is one of the key parameters to be evaluated in the detection system. The detection system is estimated through recovery additions of known amounts of the biomarker to healthy human sera as an additional evaluation approach [23]. Since the magnetic microspheres were uniform in size, they could be easily discriminated at the same location on the horizontal $x$-axis from their characterized background FSC signals (Figures 4(a) and 4(b)). The fluorescence signals of the monodisperse detected in the FL1 channel as shown on the vertical $y$-axis exhibited a clear decreasing tendency with the magnetic microspheres and coded magnetic microspheres. Herein, a series of cTnT standard solutions were analyzed to construct the fitting curves in Figure 4(c). From the changes of fluorescence signals of FITC to $\mathrm{cTnT}$ concentrations, the fitted binding response curve is plotted in Figure 4(d). The calibration equation was calculated as $Y=0.024+(0.994-0.024) /\left(1+(X / 12.095)^{1.135}\right)$. The linear detection ranges $\left(R_{10}-R_{90}\right)$ was calculated from $1.7 \mathrm{ng} / \mathrm{mL}$ to $106.1 \mathrm{ng} / \mathrm{mL}$ [23]. The accuracy evaluation experiments were conducted at five spiking levels $(2,5,10$, 20 , and $50 \mathrm{ng} / \mathrm{mL}$ ) in three duplicates for each gradient. As shown in Figure 4(e), the variation curve of binding response in the mock samples and the standard presents excellent correspondence, indicating high accuracy in the established detection method for the quantitation of cTnT.

3.5. Interference Research. The selectivity of this system should be partially attributed to the specific hybridization between the target cTnT and the antibodies on the magnetic microspheres. Therefore, different kinds of proteins were used to test the anti-interference performance and specificity of this strategy.

The binding response was used to reflect the concentration of the target in complicated conditions. Bovine serum albumin (BSA); casein; a mixture of BSA, casein, and cTnT; and cTnT were dissolved in human serum to prepare an experimental gradient. As shown in Figure 5(a), the reduction in binding reaction was significant in the presence of cTnT, and further mixtures did not affect this reduction, indicating that the reduction was caused by cTnT. The obtained BSA and casein signals had almost no targets. This hybridization does not affect coupling between cTnT in the samples or standards and rabbit anti-cTnT. In short, depending on antigen-antibody recognition and antibody-IgGFITC, this detection method showed high specificity for the target $\mathrm{cTnT}$.

3.6. Application of Magnetic Microspheres Based on the Detection Method. Our method showed pronounced antiinterference performance and high specificity. Owing to the presence of magnetic microspheres, it is easy to remove excess fluorescent probes and other autofluorescent substances in the detection system to reduce background noise [30]. Furthermore, the versatility of the flow cytometer itself could resolve extraordinary complex analytical mixtures. Since the FSC signal only depends on the size of particles, by limiting the fluorescence collection to a population with an appropriate FSC signal, the fluorescent beads can be readily distinguished from any fluorescence interference [31]. Therefore, in order to prove the feasibility of this technique, it is important to apply this system to the detection of patient serum indexes [32].

cTnT is an important biomarker in the serum of patients with myocardial infarction. Another important parameter is that the minimum fluorescence intensity collected from the 


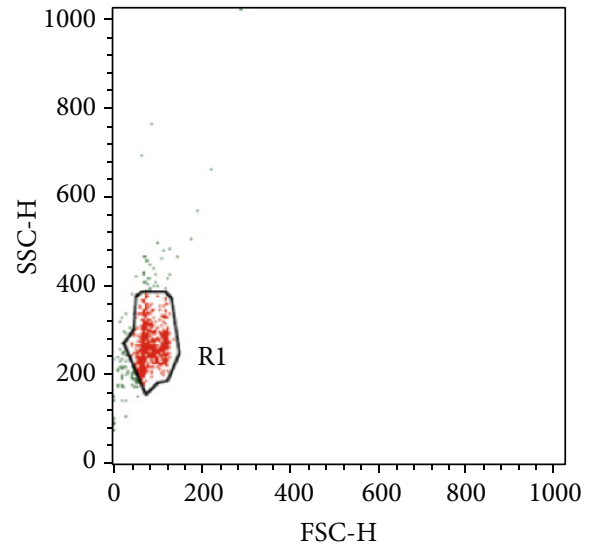

(a)

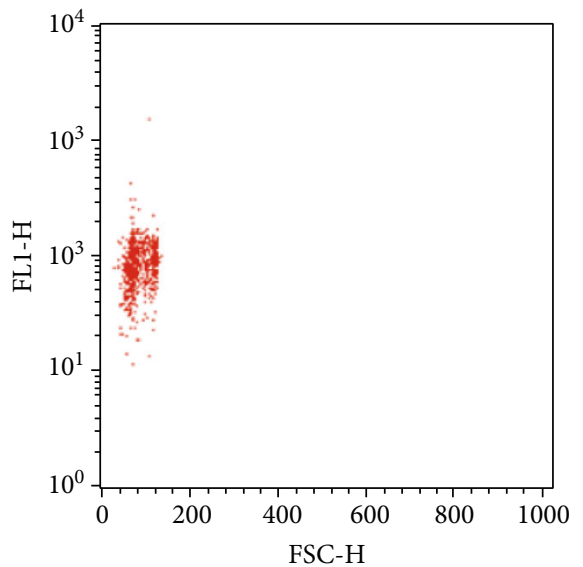

(b)

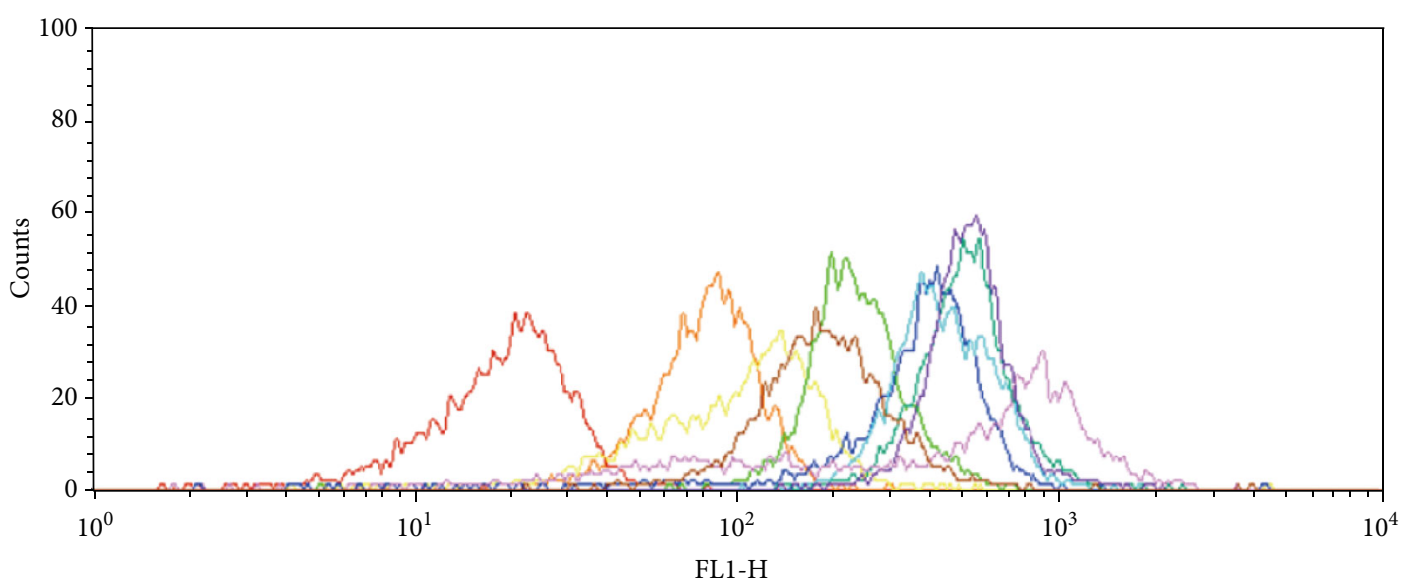

(c)

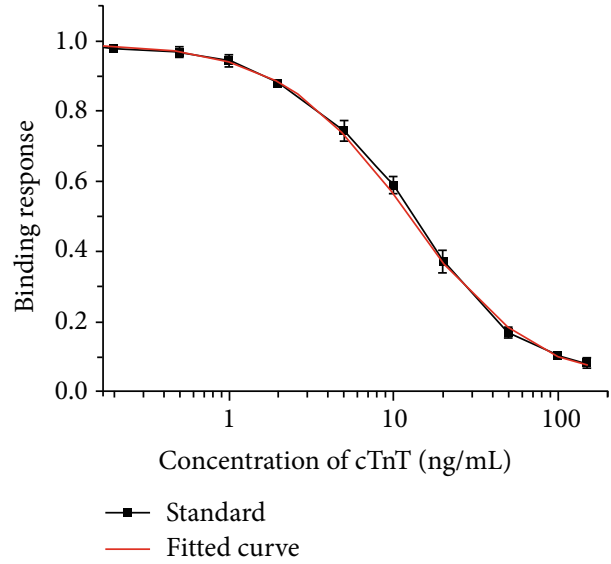

(d)

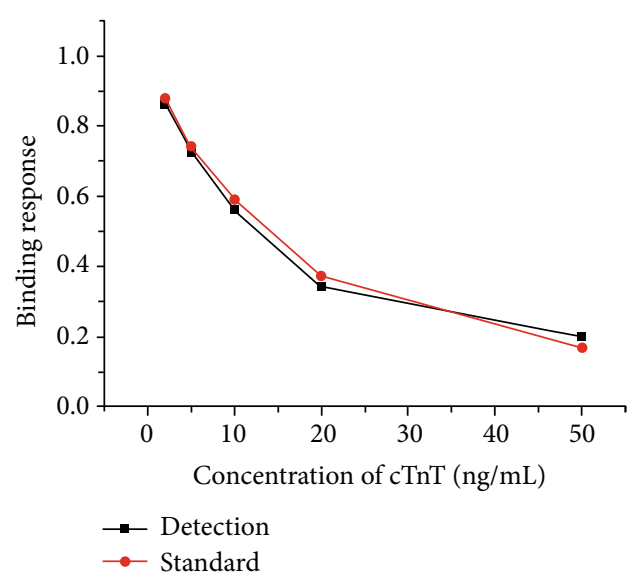

(e)

FIGURE 4: (a) Scatter plots (FSC vs. SSC) of magnetic microspheres-cTnT. (b) Scatter plots (FL1-H vs. FSC-H) of coded magnetic microspheres. (c) Diagram of fluorescence fitting curves in the presence of different concentrations of target cTnT. (d) Binding response of anti-cTnT coupled with magnetic microspheres at different concentrations of cTnT ranging from $0.1,0.2,0.5,1,2,5,10,20,50$, and 100 to $150 \mathrm{ng} / \mathrm{mL}$. (e) Assay control of detection at different concentrations of standard.

magnetic microspheres must be greater than three times the background signal [33]. Based on the combination of target cTnT with anti-cTnT, antibodies with microspheres-cTnT, and fluorescence probe encoding magnetic microspherescTnT, the fluorescence intensity could be directly collected and then converted into a binding reaction through an analytical formula. cTnT was dissolved in human sera at concentrations of $0.1,0.2,0.5,1,2,5,10,20,50,100$, and $150 \mathrm{ng} / \mathrm{mL}$ to construct our mock patient serum. The variation curve of fluorescence intensity is shown in Figure 5(b). The results 

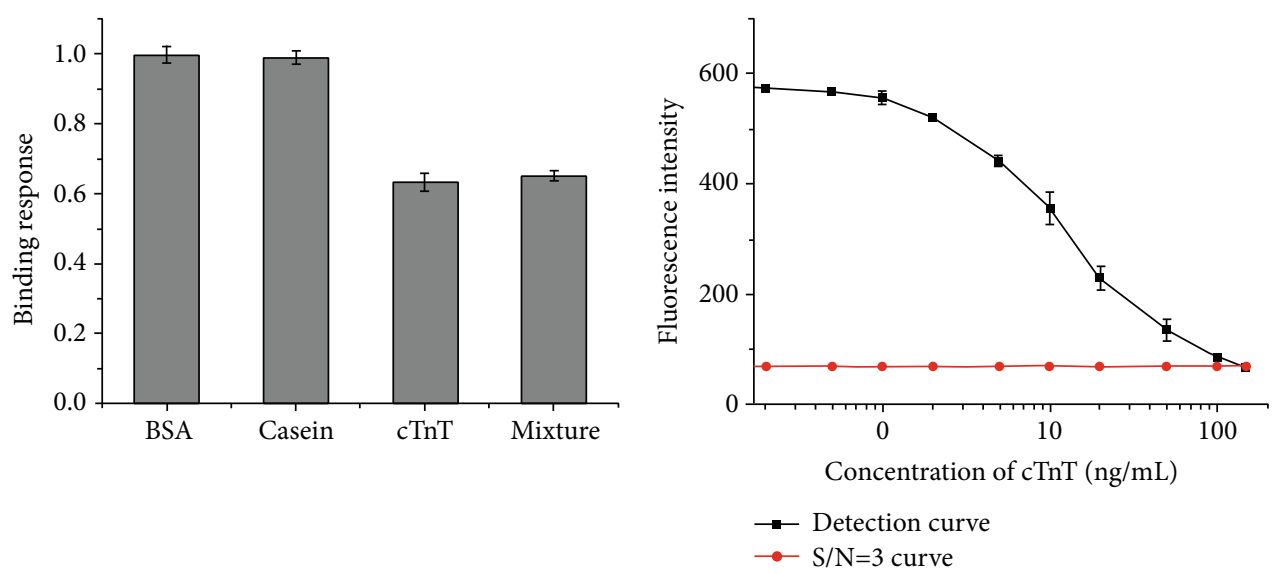

(a)

(b)

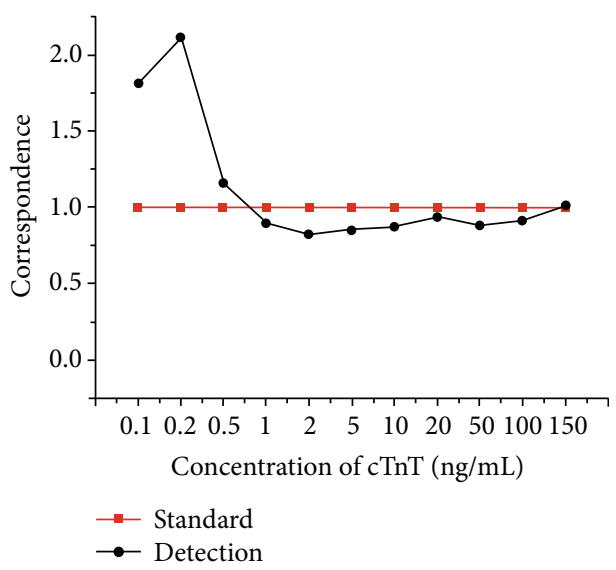

(c)

FIGURE 5: (a) Binding response histogram of different matrix resolutions (BSA, casein, a mixture, and cTnT). (b) A curve of fluorescence intensity of different concentrations of $c \operatorname{TnT}$ and a signal-to-noise ratio $(\mathrm{S} / \mathrm{N}=3)$ curve. (c) Detection correspondence and standard correspondence curves against different concentrations of cTnT.

show that this is consistent with the trend of the binding reaction curve, and the horizontal line below is a signal-tonoise ratio curve, indicating that the quantification detection range of this method was below a concentration of $150 \mathrm{ng} / \mathrm{mL}$. As shown in Figure 5(c), the corresponding curve was calculated from the detection concentration divided by the actual concentration in human sera. Therefore, points closer to the level on the graph indicate that they are approaching high specificity and accuracy. The cTnT concentrations in the range of 0.5 to $100 \mathrm{ng} / \mathrm{mL}$ showed pronounced sensing performance. Therefore, the limit of detection (LOD) is $0.5 \mathrm{ng} / \mathrm{mL}$. Furthermore, depending on the calibration equation and accuracy analysis, the limit of quantification (LOQ) is $1.7-106.1 \mathrm{ng} / \mathrm{mL}$ in our method.

\section{Conclusions}

In summary, this experiment describes and validates a novel cost-effective magnetic microsphere-based flow cytometric method for rapid quantification of cTnT. This method takes advantage of magnetic microspheres and is easy to operate. The results show high accuracy and specificity in the quantification concentration gradient of $1.7-106.1 \mathrm{ng} / \mathrm{mL}$, with a detection limit of $0.5 \mathrm{ng} / \mathrm{mL}$. Furthermore, based on the advantages of flow cytometry, this method could be applied to detect more than one type of AMI biomarker at the same time, such as cTnT, cTnI, and creatine kinase isoenzymes (CK-MB), making the early diagnosis of AMI more accurate and rapid.

\section{Data Availability}

All datasets used in this study are included in the manuscript.

\section{Conflicts of Interest}

The authors declare that there is no conflict of interest regarding the publication of this paper.

\section{Acknowledgments}

This work was supported by grants from the National Natural Science Foundation of China (81771965, 31571017, 81571791, and 81271697), the National "Significant New Drug Creation" Science and Technology Major Program (No. 2012ZX09503001-003), the Project of Department of 
Science and Technology of Jilin Province, China (No. 20170204027NY, 20130206069GX, 20200801015GH, and 20200404100YY), Jilin Province Health Technology Innovation Project (2017J042), and the Special Project of Biological Medicine of Jilin Province, China (No. SXGJSF2017-1).

\section{References}

[1] F. Li, L. Guo, Y. Hu et al., "Multiplexed chemiluminescence determination of three acute myocardial infarction biomarkers based on microfluidic paper-based immunodevice dual amplified by multifunctionalized gold nanoparticles," Talanta, vol. 207, pp. 120346-120346, 2020.

[2] Z. Zhelev, C. Hyde, E. Youngman et al., "Diagnostic accuracy of single baseline measurement of Elecsys Troponin T highsensitive assay for diagnosis of acute myocardial infarction in emergency department: systematic review and meta-analysis," $B M J$, vol. 350, 2015.

[3] R. Body, G. Burrows, S. Carley et al., "High-sensitivity cardiac troponin $\mathrm{T}$ concentrations below the limit of detection to exclude acute myocardial infarction: a prospective evaluation," Clinical Chemistry, vol. 61, no. 7, pp. 983-989, 2015.

[4] J. T. Neumann, N. A. Sörensen, F. Ojeda et al., "Immediate rule-out of acute myocardial infarction using electrocardiogram and baseline high-sensitivity troponin I," Clinical Chemistry, vol. 63, no. 1, pp. 394-402, 2017.

[5] H. Jo, H. Gu, W. Jeon et al., "Electrochemical aptasensor of cardiac troponin I for the early diagnosis of acute myocardial infarction," Analytical Chemistry, vol. 87, no. 19, pp. 98699875, 2015.

[6] S. A. Barnason, L. M. Zimmerman, J. L. Nieveen, P. S. Schulz, and L. Young, "Patient recovery and transitions after hospitalization for acute cardiac events: an integrative review," Journal of Cardiovascular Nursing, vol. 27, no. 2, pp. 175-191, 2012.

[7] T. Liu, L. Liang, P. Xiao et al., “A label-free cardiac biomarker immunosensor based on phase-shifted microfiber Bragg grating," Biosensors and Bioelectronics, vol. 100, pp. 155-160, 2018.

[8] E. P. M. Cardinaels, A. M. A. Mingels, T. van Rooij, P. O. Collinson, F. W. Prinzen, and M. P. van Dieijen-Visser, "Timedependent degradation pattern of cardiac troponin T following myocardial infarction," Clinical Chemistry, vol. 59, no. 7, pp. 1083-1090, 2013.

[9] D. Cardinale, A. Colombo, G. Bacchiani et al., "Early detection of anthracycline cardiotoxicity and improvement with heart failure therapy," Circulation, vol. 131, no. 22, pp. 1981-1988, 2015.

[10] T. Reinhold, E. Giannitsis, M. Mockel et al., "Cost analysis of early discharge using combined copeptin/cardiac troponin testing versus serial cardiac troponin testing in patients with suspected acute coronary syndrome," PLoS One, vol. 13, no. 8, article e0202133, 2018.

[11] O. Mahmoud, H. Mahmaljy, M. Youniss et al., "Comparative outcome analysis of stable mildly elevated high sensitivity troponin $\mathrm{T}$ in patients presenting with chest pain. A single-center retrospective cohort study," IJC Heart \& Vasculature, vol. 30, article 100586, 2020.

[12] A. M. A. Mingels, L. H. Jacobs, E. C. H. J. Michielsen, J. C. J. M. Swaanenburg, W. K. W. H. Wodzig, and M. van Dieijen-Visser, "Reference population and marathon runner sera assessed by highly sensitive cardiac troponin $\mathrm{T}$ and commercial cardiac troponin T and I assays," Clinical Chemistry, vol. 55, no. 1, pp. 101-108, 2009.

[13] A. V. Vylegzhanina, A. E. Kogan, I. A. Katrukha et al., "Fullsize and partially truncated cardiac troponin complexes in the blood of patients with acute myocardial infarction," Clinical Chemistry, vol. 65, no. 7, pp. 882-892, 2019.

[14] W. Schlecht and W.-J. Dong, "Dynamic equilibrium of cardiac troponin C's hydrophobic cleft and its modulation by $\mathrm{Ca} 2+$ sensitizers and a $\mathrm{Ca} 2+$ sensitivity blunting phosphomimic, cTnT (T204E)," Bioconjugate Chemistry, vol. 28, no. 10, pp. 2581-2590, 2017.

[15] J. Boeddinghaus, T. Nestelberger, R. Twerenbold et al., "Highsensitivity cardiac troponin I assay for early diagnosis of acute myocardial infarction," Clinical Chemistry, vol. 65, no. 7, pp. 893-904, 2019.

[16] E. Samaha, J. C. Brown, F. Brown et al., "High-sensitivity cardiac troponin T increases after stress echocardiography," Clinical Biochemistry, vol. 63, pp. 18-23, 2019.

[17] T. Reichlin, W. Hochholzer, S. Bassetti et al., "Early diagnosis of myocardial infarction with sensitive cardiac troponin assays," The New England Journal of Medicine, vol. 361, no. 9, pp. 858-867, 2009.

[18] Q. Wang, Y. Lei, X. Lu et al., "Urea-mediated dissociation alleviate the false-positive Treponema pallidum-specific antibodies detected by ELISA," PLoS One, vol. 14, no. 3, article e0212893, 2019.

[19] L. C. V. Alves, M. D. G. Carvalho, F. F. C. Nunes et al., "Evaluation of potential biomarkers for the diagnosis and monitoring of systemic lupus erythematosus using the cytometric beads array (CBA)," Clinica Chimica Acta, vol. 499, pp. 1623, 2019.

[20] A. D. S. Moraes, D. G. Brum, J. C. M. Ierich et al., "A highly specific and sensitive nanoimmunosensor for the diagnosis of neuromyelitis optica spectrum disorders," Scientific Reports, vol. 9, no. 1, article 16136, 2019.

[21] C. Haferlach, S. O. Twardziok, S. Hutter et al., "Whole genome sequencing identifies single nucleotide variants, structural and copy number abnormalities with a high sensitivity in hematological neoplasm: an all in one diagnostic tool tested on 3241 cases," Blood, vol. 132, Supplement 1, pp. 15251525, 2018.

[22] M. R. Newman, B. J. Blyth, D. J. Hussey, D. Jardine, P. J. Sykes, and R. J. Ormsby, "Sensitive quantitative analysis of murine LINE1 DNA methylation using high resolution melt analysis," Epigenetics, vol. 7, no. 1, pp. 92-105, 2012.

[23] W. Kong, C. Xiao, G. Ying et al., "Magnetic microspheresbased cytometric bead array assay for highly sensitive detection of ochratoxin A," Biosensors and Bioelectronics, vol. 94, pp. 420-428, 2017.

[24] X. He, C. Liang, Q. Liu, and Z. Xu, "Magnetically responsive Janus nanoparticles synthesized using cellulosic materials for enhanced phase separation in oily wastewaters and water-incrude oil emulsions," Chemical Engineering Journal, vol. 378, article 122045, 2019.

[25] L. Zhang, X. Yue, N. Li et al., "One-step maltosefunctionalization of magnetic nanoparticles based on selfassembled oligopeptides for selective enrichment of glycopeptides," Analytica Chimica Acta, vol. 1088, pp. 63-71, 2019.

[26] S. Z. Mazlan, F. Rahim, and S. Abu Hanifah, "Facile synthesis of magnetic copolymer microspheres based on poly(glycidyl methacrylate-co-N-isopropylacrylamide)/Fe3O4by 
suspension photopolymerization," International Journal of Polymer Science, vol. 2014, Article ID 591898, 7 pages, 2014.

[27] L. Zheng, G. Cai, S. Wang, M. Liao, Y. Li, and J. Lin, "A microfluidic colorimetric biosensor for rapid detection of Escherichia coli O157:H7 using gold nanoparticle aggregation and smart phone imaging," Biosensors and Bioelectronics, vol. 124-125, pp. 143-149, 2019.

[28] Y. Chen, R. Medhi, I. Nekrashevich, D. Litvinov, S. Xu, and T. R. Lee, "Specific detection of proteins using exceptionally responsive magnetic particles," Analytical Chemistry, vol. 90, no. 11, pp. 6749-6756, 2018.

[29] Y. Wang, F. Zhang, H. Lin, and F. Qu, "Biodegradable hollow $\mathrm{MoSe} 2 / \mathrm{Fe} 3 \mathrm{O} 4$ nanospheres as the photodynamic therapyenhanced agent for multimode CT/MR/IR imaging and synergistic antitumor therapy," ACS Applied Materials \& Interfaces, vol. 11, no. 47, pp. 43964-43975, 2019.

[30] B. K. Parida, H. Garrastazu, J. K. Aden, A. P. Cap, and S. J. Mcfaul, "Silica microspheres are superior to polystyrene for microvesicle analysis by flow cytometry," Thrombosis Research, vol. 135, no. 5, pp. 1000-1006, 2015.

[31] W. Chang, H. Shang, R. Perera et al., "Rapid detection of dengue virus in serum using magnetic separation and fluorescence detection," Analyst, vol. 133, no. 2, pp. 233-240, 2008.

[32] J. Kim, M. J. Biondi, J. J. Feld, and W. C. W. Chan, "Clinical validation of quantum dot barcode diagnostic technology," ACS Nano, vol. 10, no. 4, pp. 4742-4753, 2016.

[33] J. Proust, J. P. Martin, D. Gérard, J. L. Bijeon, and J. Plain, "Detecting a zeptogram of pyridine with a hybrid plasmonic-photonic nanosensor," ACS Sensors, vol. 4, no. 3, pp. 586-594, 2019. 